\title{
La investigación en Comunicación ante una encrucijada: de la teoría de los campos a la diseminación y diversidad gnoseológica. Estudio inicial comparado entre España, Brasil y Chile ${ }^{1}$
}

\section{Víctor Silva Echeto² Jordi de San Eugenio Vela ${ }^{3}$}

Recibido: 2013-11-09

Envío a pares: 2013-11-12
Aprobado por pares: 2014-02-05

Aceptado: 2014-02-20

DOI: $10.5294 /$ pacla.2014.17.3.10

Para citar este artículo / To reference this article / Para citar este artigo

Silva, V., San Eugenio, J. Septiembre de 2014. La investigación en Comunicación ante una encrucijada: de la teoría de los campos a la diseminación y diversidad gnoseológica. Estudio inicial comparado entre España, Brasil y Chile. Palabra Clave 17 (3), 803-827. DOI: 10.5294/pacla.2014.17.3.10

\section{Resumen}

Desde sus inicios, la comunicación fue un espacio de investigación diverso, complejo, atravesado por múltiples influencias (sociológicas, políticas, económicas y semióticas). En ese contexto, la teoría de los campos (apuntalada por Pierre Bourdieu) brindó las posibilidades de estructuración y de autonomía, como mecanismo de reducir esa diversidad. Sin embargo, en estos años la heteronomía del campo, ha conducido a la comunicación

1 Este artículo forma parte del proyecto financiado por el Fondo Nacional de Desarrollo Científico y Tecnológico, Ministerio de Educación, Gobierno de Chile (Fondecyt) 1130921 "Análisis crítico y complejo del discurso periodístico verbovisual de la representación intercultural limítrofe/liminal en la prensa boliviana, chilena y peruana sobre el diferendo marítimo internacional de La Haya”, dirigido por el Dr. Rodrigo Browne Sartori.

2 Universidad de Playa Ancha, Chile. vsilva@upla.cl

3 Universidad de Vic-Universidad Central de Cataluña, España. jordi.saneugenio@uvic.cat 
(como en el caso del arte u otras disciplinas) a influenciarse por contextos globales, inter y transdisciplinarios e intermediales (o transmediales), por tanto, no son los medios su objeto de investigación en sí mismos, sino una diversidad gnoseológica. En el artículo, se pone en discusión estos aspectos teóricos, contrastándolos con un estudio comparado -inicial- sobre la investigación en comunicación en España, Brasil y Chile, tres de los países con un mayor desarrollo en los últimos años.

\section{Palabras clave}

Investigación, comunicación, España, Brasil, Chile (Fuente: Tesauro de la Unesco).

\section{A Dilemma in Communication Research: From the Field Theory to Gnoseological Diversity and Dissemination. An Initial Study Comparing Spain, Brazil and Chile}

\section{Abstract}

From its onset, communication has been a diverse and complex area of investigation, influenced by many fields (sociology, politics, economics, technology, and semiotics). In this context, the field theory (supported by Pierre Bourdieu) offers different possibilities for organization and autonomy as a way to reduce that diversity. However, at this point, the heteronomy of the field has led to a situation where communication (as with art or other disciplines) is being influenced by global, interdisciplinary, transdisciplinary, and intermedial (or transmedial) contexts. Hence, it is gnoseological diversity that is the object of investigation, not the mass media itself. These theoretical aspects are discussed in this article and weighed against an initial comparative study on investigation in communication in Spain, Brazil and Chile, which are three of the countries with the most development in the last few years. 


\section{Keywords}

Research, communication, Spain, Brazil, Chile (Source: UNESCO Thesaurus).

\section{A pesquisa em Comunicação ante uma encruzilhada: da teoria dos} campos à disseminação e diversidade gnoseológica. Estudo inicial comparado entre Espanha, Brasil e Chile

\section{Resumo}

Desde seu início, a comunicação foi um espaço de pesquisa diverso, complexo, que recebeu múltiplas influências (sociológicas, políticas, econômicas e semióticas). Nesse contexto, a teoria dos campos (sustentada por Pierre Bourdieu) ofereceu as possibilidades de estruturação e de autonomia, como mecanismo de redução dessa diversidade. Contudo, nesses anos, a heteronímia do campo conduziu a comunicação (como no caso da arte ou de outras disciplinas) a ser influenciada por contextos globais, inter e transdisciplinares e intermediais (ou transmediais); portanto, não são os meios seu objeto de pesquisa em si mesmos, mas sim uma diversidade gnoseológica. Neste artigo, põe-se em discussão esses aspectos teóricos contrastando-os com um estudo comparado — inicial— sobre a pesquisa em comunicação na Espanha, Brasil e Chile, três dos países com maior desenvolvimento nos últimos anos.

\section{Palavras-chave}

Pesquisa, comunicação, Espanha, Brasil, Chile (Fonte: Tesauro da Unesco). 


\section{Introducción: la teoría de los campos}

El giro teórico-conceptual de la segunda mitad del siglo XX implicó una transformación radical en la 'episteme' (Foucault, 1986) occidental y en su extensión latinoamericana. Entre ellas se produjo una 'mutación’ epistemológica, donde las disciplinas como mecanismos de compartimentar los saberes son puestas en cuestionamiento por cruces entre ámbitos del conocimiento que, en las primeras décadas del siglo XX y en las últimas del XIX, habían sufrido la llamada 'vigilancia' epistemológica como forma de mantener esa separación entre disciplinas (consideradas como ciencias), artes y filosofía. La comunicación, en ese contexto, surge como área de conocimiento e intenta consolidarse en esa época (Marques de Melo, 2007). Es, por tanto, parte de la transformación, pero, también, de la crisis.

Una de las propuestas más innovadoras es la teoría de los campos de Pierre Bourdieu, que, aunque mantiene la vigilancia sobre las estructuras del saber, paralelamente permite flexibilizar sus límites. Si el campo presenta cierta 'autonomía' como espacio de saber y de hacer, a su vez, su interior es el lugar donde se producen las 'luchas simbólicas'.

En un comienzo la teoría de los campos se ubica al interior de la 'sociología', considerando los 'campos sociales' como estructuras objetivas, en cuyo interior se producen y reproducen las 'luchas simbólicas' propiciadas por diversas formas de 'violencia' (el sistema educativo, por ejemplo, como mecanismo de reproducción de esa violencia semiótica). Pero la teoría de los campos puede, a su vez, extenderse al campo científico y a su ansiada autonomía. Ésta es una de las obsesiones -no siempre comprendidas- del sociólogo francés, porque la insistencia por la autonomía lo lleva a referirse a diversos espacios autónomos vinculados entre sí por una zona punteada o de fronteras que, a su vez, lleva a que cada uno de ellos conserve cierta independencia.

Con referencia a la autonomía en el campo científico, para Bourdieu (2000, p. 76), cuanto más autónomo sea el campo, "más intenso será su poder de refracción y más se transfigurarán las coacciones externas, al extremo de volverse, con frecuencia, absolutamente, irreconocibles”. Su 
grado de autonomía, "en consecuencia, tiene como indicador principal su poder de refracción y retraducción". Inversamente, "la heteronomía de un campo se manifiesta esencialmente en el hecho de que los problemas exteriores, en especial, los políticos se expresan directamente en él". El temor de Bourdieu, por eso su insistencia en la autonomía, es la 'politización' del campo científico. Al respecto, "una de las grandes dificultades con la que se toman las ciencias sociales (...) es el hecho de que personas poco competentes, desde el punto de vista de las normas específicas, siempre puedan intervenir en nombre de principios heterónomos sin que se las descalifique de inmediato".

Bourdieu escribió que la dificultad de las ciencias sociales (donde generalmente se ubica a la comunicación que, en algunas corrientes, nace al interior de la sociología como sociología de la comunicación) frente a las otras ciencias (llamadas de la 'naturaleza') es que éstas podían obviar la discusión sobre la objetividad o la subjetividad del científico, mientras que en las ciencias sociales aparece constantemente la discusión sobre la 'mirada del científico' (llamado en muchos momentos despectivamente cientista). La propuesta de Bourdieu, en ese sentido, era historizar al sujeto que conoce, objetivar al sujeto de la objetivación, esto es, lo trascendental histórico, cuya objetivación era la condición de acceso de la ciencia a la conciencia de sí, o sea, al conocimiento de sus presupuestos históricos (Bourdieu, 2000).

La sociología de la ciencia, por tanto, reposa en el postulado de que la verdad del producto (la llamada verdad cientifica) reside en particulares condiciones sociales de producción; es decir, en un estado determinado de la estructura y del funcionamiento del campo científico. Esta concepción, planteada por Bourdieu, sigue los pasos de Thomas Kuhn, para quien "una comunidad de especialistas (de ciencia) se esmerará por asegurarse la progresión en la acumulación de datos que ella pueda usar con precisión y con detalle" (Kuhn, 1981, p. 168). Bourdieu desmitifica al 'campo científico' considerando que en él -como en el campo social- se producen relaciones de fuerza, monopolios, luchas y estrategias, pero -a diferencia del campo social- "todas estas invariantes revisten formas específicas", como las ideas (los saberes) y las instituciones (campo científico) que les dan un 
soporte efectivo (las universidades y los institutos de investigación) (Bourdieu, 2000, p. 72). En estas se producen y difunden los papers, se dictan las conferencias, se escriben los ensayos, se participa en congresos, se investiga y se jerarquiza académicamente. Son, por todo lo indicado, las instancias de legitimación y de ritualización del campo científico o, en otros términos, 'los dispositivos' de poder.

En resumen, el campo es un espacio "relativamente" autónomo, un "microcosmos provisto de sus propias leyes", un "mundo social" en el que se ejercen coacciones, solicitaciones, "pero son relativamente independientes de las coacciones del mundo social que las engloba” (Bourdieu, 2000, p. 74). Lo que justifica, para el sociólogo, tal saber, no es un valor filosófico o moral, sino su estructuración en una comunidad científica. La complejidad que se les presenta a las ciencias sociales para mantener su autonomía es que desde sus inicios compartieron la explicación de los fenómenos sociales, es decir, no tuvieron el monopolio sobre él. Al principio lo compartieron con la filosofía y la teología, posteriormente, con el periodismo (Silva y Browne, 2007).

La idea de campo científico, por tanto, integra un nuevo dato a la discusión sobre el estatuto de las ciencias sociales. El ejercicio de la crítica social, para la teoría de los campos, sólo puede ejercerse planteándose desde la historicidad de las prácticas sociales y localizándose en un lugar específico.

El campo es ese territorio, "lugar jerarquizado, estructurado según una determinada lógica de intereses”. En él se agrupa, se integra, se complementa e ingresa en conflicto un grupo específico de actores: los científicos. A estos agentes se les reconoce la capacidad de hablar e intervenir legítimamente (de manera autorizada y con autoridad) en materia de ciencia. Los juicios sobre las capacidades científicas están siempre contaminados por el conocimiento de la posición que ocupan en las jerarquías instituidas. El papel del agente (científico, periodista, maestro) en la teoría de los campos es fundamental; no obstante, no es una perspectiva epistemológica más en las teorías sociológicas sobre la acción, ya que, en aquel caso, juega un papel fundamental la estructuración del habitus de los agentes, es decir, tanto 
las estructuras estructuradas (cuando ya llega al campo) como las estructurantes (las que el agente puede ir cambiando).

En este punto, resulta interesante recuperar el planteamiento de Arias y Roncallo (2012), quienes proponen un viaje de la epistemología a la política, con el fin de repensar la reflexión en torno al campo de la comunicación. El contexto posmoderno parece cuestionar la tradicional dicotomía entre unidad-fragmentación del campo; una apuesta, además, por una definitiva huida de las 'verdades absolutas' (paradigmas) para depositar el énfasis en el contexto, en el territorio de origen en el que se produce el análisis del campo de la comunicación. Arias y Roncallo (2012, p. 216) se pronuncian en los siguientes términos: "El problema de un campo como la comunicación no es decidir si este continúa basándose en una narrativa unitaria o si ha sucumbido a la inevitable fragmentación de nuestro tiempo. El problema real es qué tipo de unidades son producidas dentro y entre los 'fragmentos' particulares, y cómo estas unidades son construidas".

Por tanto, la unidad y la fragmentación del campo de la comunicación deben entenderse en grado de consubstancialidad, de normalidad $y$, tal vez, el énfasis deba ahora trasladarse a la naturaleza de la pregunta en torno al campo que resulta propio al estudio de la comunicación. En esta línea, Arias y Roncallo (2012) sugieren una pregunta en torno a las condiciones a partir de las cuales se produce una idea particular de unidad: qué tipo de unidades narrativas se producen y por qué. La respuesta a esta pregunta plantea un interesante viaje de la epistemología (objeto de la investigación) a la política (resultado de una determinada forma de preguntar), entendiendo el término política como un proceso de producción de subjetividad, como 'lo mío y lo tuyo', priorizando, en cualquier caso, el contexto para el cuestionamiento de la universalización del campo de la comunicación.

En esta tesitura, y en el campo concreto de la investigación en comunicación, la visión predominante (angloamericana) impediría una imprescindible abstracción del contexto, con el fin de evitar la subordinación de discursos provenientes de la alteridad, en beneficio de una visión hegemónica y subjetiva del conocimiento. 
Por tanto, y siguiendo a Arias y Roncallo, se impone la

necesidad que el campo incremente su reflexión acerca de sus modos singulares de preguntar por un objeto y de indagar sobre sí mismo. (...) El campo necesita pensar esta permanente dialéctica entre unidad y fragmentación, es decir, reflexionar acerca de qué tipo de narrativas son creadas cuando se plantea la pregunta por el estatus del campo, usando unos términos en lugar de otros (2012, p. 226).

La auténtica 'ciencia de la ciencia' sólo puede constituirse rechazando la posición abstracta entre un análisis inmanente interno y un análisis externo que relaciona sus problemas con sus condiciones sociales de aparición (tal como lo planteó el marxismo).

Para Pierre Bourdieu (2000), el grado de autonomía determina, a su vez, el grado de arbitrariedad. Ésta, tomada de la semiótica saussureana, es una constante en la sociología de Bourdieu, y alcanza su cima teórica con su consideración de lo social como 'arbitrario cultural'. Al interior de un espacio social determinado la autonomía del campo es un lugar fronterizo, es decir, delimitación de un espacio en contraposición a otros espacios. Las fronteras son porosas, y se vinculan con la noción de Bachelard (1972) de ruptura epistemológica, un artificio que instaura el discurso científico en relación con el sentido común.

En lo referido a la metodología, se ha partido de un análisis de fuentes documentales (Dulzaides y Molina, 2004) que ha servido para fijar las bases de un análisis comparativo de la investigación de la comunicación en España, Brasil y Chile. Asimismo, se ha utilizado la técnica de la entrevista en profundidad para complementar los datos recabados en la fase documental de la presente investigación.

El presente artículo se estructura a partir de una introducción sustentada en la teoría de los campos. A continuación, se propone una revisión específica de la vinculación entre los términos campo y comunicación. El cuestionamiento acerca de la unidad o fragmentación del campo de la comunicación traslada la lectura hacia el debate en términos de disciplina, indisciplina o transdisciplina comunicativa. Por último, la investigación 
propone un estudio comparado inicial en lo relacionado con la investigación de la comunicación en España, Brasil y Chile. Una propuesta de discusión y algunas reflexiones finales (conclusiones) cerrarán la disposición de contenidos de este trabajo.

\section{La comunicación como campo: auge y crisis}

En España, la teoría de los campos llegó tardíamente a la comunicación. Inicialmente estaba más vinculada a la sociología de la comunicación de corte funcionalista o frankfurtiano o a la semiótica de la comunicación, como sostiene, entre otros autores, Miquel de Moragas Spà (2011). En América Latina, no obstante, encontró recepción temprana en teóricos como Néstor García Canclini o Renato Ortiz, vinculados a los llamados estudios culturales latinoamericanos (Silva y Browne, 2007).

La teoría de los campos permitía al científico encontrar un punto intersticial entre la sociología y el arte, y ese punto intermedio era la comunicación. En términos de García Canclini (2011, p. 32), "Bourdieu no fue el primero en advertir que uno de los rasgos de la modernidad era la constitución de campos autónomos (...) pero fue el sociólogo que construyó una teoría más sofisticada y rigurosa sobre las maneras” en que el arte o las ciencias se separaron de sus condicionamientos externos. Más en el caso de la comunicación, donde la industria ha estado, desde los inicios científicos de aquélla, vinculada a sus programas de investigación, influenciando en la pérdida de autonomía del campo de la comunicación. Por tanto, la autonomía implicaba ese espacio de investigación en conflicto con esos condicionamientos que marcan, de manera fundamental, a la comunicación en el capitalismo tardío.

No obstante, en fechas recientes se ha puesto en cuestionamiento la teoría de los campos, criticando su afán de autonomía, situación no asimilable en la era de la posautonomía. Es decir, en el capitalismo tardío se producen cruces y contaminaciones múltiples entre lo político, lo social y lo económico, y aún más en la comunicación. Lo posautónomo se refiere a los procesos en los que se producen 'desplazamientos de las prácticas' comunicativas basadas en medios (la tradicional separación de medios de 
comunicación que hasta no hace mucho estuvo presente tanto en los currículos docentes como en los programas de investigación) al insertarse las imágenes y los discursos en los "espacios urbanos, redes digitales y formas de participación social” (García Canclini, 2011,p. 17). Comunicación y desarrollo, comunicación y economía política, comunicación y cultura y un conjunto de posibilidades muy amplias que ha abierto la tecnología permiten cuestionar, actualmente, la teoría de los campos y su ansiada autonomía. La independencia y autocontención de las prácticas científicas, que delimitaban quiénes tenían legitimidad para decir qué era ciencia, es decir, los científicos, se ha desvanecido. Más aún en el caso de la comunicación. Para algunos teóricos (García Canclini, 2011, p. 40), "estamos en medio de un giro transdisciplinario, intermedial y globalizado”.

\section{¿Disciplina, indisciplina o transdisciplina en comunicación?}

Son muchos los investigadores que coinciden en señalar la indisposición de un estatuto disciplinar en comunicación (Pereira, 2005; Silva y Browne, 2007; Fuentes, 2008; Rizo, 2012), aunque también existen posicionamientos en favor de una doble vía: campo de estudio más disciplina, con algunos matices (Rodrigo, 2001; Piñuel, 2010; Moragas, 2011). De igual forma, cada vez son menos las manifestaciones partidarias del carácter únicamente disciplinar de la comunicación. Asimismo, Craig (1999) sostiene la posibilidad de unificación de los teóricos de la comunicación mediante lo que él denomina como 'diálogo', que supone, en opinión de este autor, una "tensión dialéctica y dialógica" entre las diferentes tradiciones existentes en comunicación.

Por su parte, Donsbach (2006) mantiene la existencia de tres pronunciamientos principales en torno a la identidad de la investigación en comunicación. En primer lugar, se refiere al enorme crecimiento experimentado en los últimos treinta años por la investigación en comunicación. El crecimiento, sin embargo, no es sinónimo de fortalecimiento disciplinar, sino de una clara pérdida de identidad de la investigación, según manifiesta el propio autor. La segunda tesis pone de relieve una meridiana acumulación de evidencias empíricas sobre el proceso de la comunicación. Ello implica una erosión epistemológica del campo, en palabras del propio autor. Por 
último, Donsbach (2006) se refiere a la proliferación de un conocimiento de la comunicación ciertamente preciso, aunque carente de orientación normativa en la investigación empírica.

Fuentes (2012) 4 afirma que la comunicación, per se, es un campo de estudio, es por ello que la comunicación entendida como disciplina se concibe, únicamente, por su necesidad de institucionalización, de posicionamiento en un marco científico, de creación de un currículo de estudios universitarios o bien de creación de colectivos de investigación que circunden un objeto concreto de estudio, entre otras razones.

Por tanto, podemos convenir que, de algún modo, la comunicación se encuentra en una encrucijada epistemológica, a razón de su marcado carácter social e interdisciplinar. En este sentido, Del Rey Morató $(1997)^{5}$ se pronuncia a favor de una epistemología de la complejidad para el campo fenomenológico de la comunicación social. Se trata de una propuesta que huye del anquilosamiento propio que se deriva de la autonomía científica disciplinar, para así ocuparse de definir un nuevo estatuto epistemológico para la comunicación: el de la interciencia. El debate de la interciencia traslada la discusión hacia la consideración de la comunicación como campo académico dentro de un ámbito de conocimiento que pivota en torno a tres posicionamientos fundamentales (Sheperd, 1993; Rodrigo, 2001; Pereira, 2005):

a. Comunicación como indisciplina-transdisciplina-posdisciplina: la comunicación implica la hibridez de saberes, el mestizaje, y, por tanto, se impone un ámbito de conocimiento que transcurre entre disciplinas, $a$ través de ellas e inclusive más allá de la propia disciplina.

b. Comunicación como antidisciplina: es la negación y la postura enfrentada a lo que hoy conocemos como disciplina. La condición posmoderna conlleva la práctica negación de las verdades absolutas y, por tanto, también de los posicionamientos inamovibles.

4 Véase más información en el siguiente documento audiovisual: http://vimeo.com/36847992.
5 Citado por Rodrigo (2001, p. 12). 
c. Comunicación como disciplina: en función de su necesidad de institucionalización. Por su carácter ontológico y simbólico (Sheperd, 1993).

\section{¿Qué se investiga en comunicación? Estudio comparado inicial: España, Brasil y Chile}

En este apartado se propondrá, como vía inicial de indagación, un estudio comparado de algunas de las temáticas junto a la inclusión de unos primeros porcentajes sobre la investigación en comunicación en tres países importantes para el área de conocimiento: España, Brasil y Chile. Se entiende que son datos todavía precarios, pero considerando las dificultades para obtener datos más acabados, la heterogeneidad de las agencias de investigación en cada uno de los países y la escasa consolidación de la comunicación, se plantea como un estudio inicial que será un primer paso para una investigación más consolidada en los próximos años. Más allá de lo expuesto, parece oportuno este primer avance para intentar la progresiva consolidación de la comunicación como ciencia social. También se alerta que el presente trabajo utiliza materiales diversos y heterogéneos, que son los que se pueden ir rastreando entre investigadores y agencias de investigación.

\section{La investigación de la comunicación en España}

Tal y como señala Moragas (2011, p. 17), los estudios de comunicación deben ofrecer claves de interpretación de la sociedad global en la que nos encontramos sumidos (cultura, política, economía). Ello supone, según el mismo autor, partir de conocimientos parciales. La parcialidad o, por qué no decirlo, la superficialidad en el tratamiento de los diferentes objetos de estudio vinculados a la comunicación, ha supuesto, por un lado, un amplio abanico temático con el cual trabajar, aunque, por el otro, ha generado a nuestro entender, y de acuerdo con Martínez-Nicolás (2009), una manifiesta falta de identidad epistemológica, teórica y metodológica relacionada con la investigación científica de la comunicación en España.

Este posicionamiento colisiona, en cierto modo, con el punto de vista defendido por García-Jiménez (2007), que argumenta una notable consolidación de la epistemología de la comunicación en España, de sus inves- 
tigadores y de las instituciones, en beneficio de un análisis más empírico y menos instrumental del campo de la comunicación.

En las líneas que siguen, proponemos un sucinto recorrido histórico y temático con voluntad crítica e interpretativa vinculado a la investigación (publicada) de la comunicación en España.

Cáceres y Caffarel (1992 y 1993) documentan los inicios de la investigación comunicativa en España. El trabajo de ambas autoras se sitúa temporalmente en lo que Martínez-Nicolás (2009) denomina como etapa de emergencia, esto es, el surgimiento de la comunicación como campo disciplinar (1965-1980). Esta etapa coincide con la institucionalización universitaria de la comunicación ${ }^{6}$ como punto de partida de la producción científica española en comunicación. A nivel temático, los tópicos de investigación que ocupan a la comunidad científica entre los años 1978 y 1990 se dirigen, claramente, hacia el estudio de los medios de comunicación de masas, ${ }^{7}$ en compañía de un inusual interés por la epistemología de la comunicación (Cáceres y Caffarel, 1993; De Aguilera, 1998). En el seno de la comunidad científica se vislumbra una primera fractura doctrinal: la que separa, en palabras de Martínez-Nicolás (2009), a practicistas ${ }^{8}$ de teoricistas. ${ }^{9}$

En esta etapa incipiente de investigación de la comunicación en España, los referentes teórico-conceptuales se encuentran en la publicística, la Escuela de Frankfurt, la mass communication research o la semiótica (Martínez-Nicolás, 2009; Martínez-Nicolás y Saperas, 2011). A nivel metodológico, se impone el despliegue de técnicas descriptivo-analíticas, las cuales acaban condicionando la propia investigación, de modo que, en algunos casos, los

6 Coincide con la impartición (curso 1971-1972) de estudios universitarios de comunicación en la Universidad Complutense de Madrid, la Universitat Autònoma de Barcelona y la Universidad de Navarra. A principios de los ochenta se añadiría la Universidad del País Vasco (Jones, 1998; Martínez-Nicolás, 2009; Martínez-Nicolás y Saperas, 2011).

7 Estudios sobre la prensa escrita, la televisión, las representaciones sociales o las políticas de comunicación, entre otros temas de interés preferente.

8 Se corresponde con la práctica incorporación a las universidades españolas de perfiles que comparten su actividad profesional (especialmente en el ámbito periodístico) con la docencia universitaria.

9 Claramente significados en dos frentes: por un lado, los denominados comunicólogos, empeñados en parcelar, autónomamente, el ámbito disciplinar de la comunicación, y, por el otro, los humanistas, con formación principalmente en historia y semiótica, quienes proporcionan un enfoque de cultura de masas a la investigación de la comunicación en España (Martínez-Nicolás, 2009). 
investigadores adaptan sus trabajos en función de la metodología utilizada, lo que sin duda habla de las deficiencias, en lo que a método se refiere, de la primeriza investigación comunicativa en España (Cáceres y Caffarel, 1993). Asimismo, cabe destacar que la sociología de la comunicación es preponderante en la ubicación disciplinar de la investigación comunicativa, seguida a distancia por la ciencia política y la teoría de la comunicación, entre otros ámbitos a destacar.

Entre los años 1980 y 1995 se produjo una evolución acelerada de la investigación comunicativa en España, íntimamente vinculada a la multiplicación de la oferta formativa superior en comunicación (De Aguilera, 1998). Martínez-Nicolás (2009) se refiere a una etapa de consolidación acompañada de una cierta explosión en cuanto a oportunidades institucionales, a la vez que una cierta agitación referida a una actualización (que no debe confundirse con equiparación) de la investigación española con la investigación anglosajona. Es el momento en que los primeros titulados en comunicación se incorporan al ámbito de la investigación. En todo caso, y tal como señala de nuevo Martínez-Nicolás (2009), se mantiene un claro enfrentamiento entre los profesionalistas y los teoricistas.

En lo referido a las tendencias de investigación, priman los trabajos que dan cuenta del ejercicio profesional de la comunicación en sus diversos ámbitos (especialmente el periodismo, aunque también la publicidad, las relaciones públicas y la comunicación audiovisual). La investigación bibliométrica llevada a cabo por Castillo y Xifra (2006) para el ámbito de las tesis doctorales españolas sobre relaciones públicas entre los años 1965 y 2005 así lo certifica. En concreto, y en cuanto a temáticas, el 72,31\% de los trabajos doctorales leídos se ocuparon de la práctica/aplicación de las relacionas públicas. A distancia, se ubica la implantación de programas y campañas (29,23\%). Es lo que Martínez-Nicolás (2009) ha denominado profesionalismo académico.

Sin embargo, la reincidencia en este tipo de estudios, enfocados desde un punto de vista de inventario (presentación de datos en muchos casos carentes de contexto e interpretación), esto es, estados de la cuestión, en detrimento de un análisis en profundidad que permita determinar por qué 
la cuestión está en ese estado (Martínez-Nicolás, 2006), manifiesta la pervivencia de un lastre que la investigación de la comunicación en España incorpora desde sus inicios: una clara carencia formativa en teoría social y en metodología de la investigación social empírica (Martínez-Nicolás, 2009).

La tercera etapa de la investigación comunicativa en España parte del año 1995 y llega hasta nuestros días. La posmodernidad, el pensamiento disruptivo, lo efímero y el presunto derribo de las verdades absolutas dan paso a una hibridez de conocimientos y a un mestizaje disciplinar harto conocido en el ámbito de la comunicación. El escenario digital, las nuevas pantallas y el universo multimedia, en un contexto de convergencia mediática, señalan nuevas posibilidades para la agenda de la investigación comunicativa en España. Asimismo, se produce el desembarco de investigadores procedentes de las ciencias sociales clásicas (economía, psicología o sociología, entre otras) aspecto que, sin duda, contribuye a la mejora del déficit metodológico y teórico-disciplinar del cual aún hoy adolece la investigación española de la comunicación.

En esta etapa, merece especial mención la notable mejora de los procesos de evaluación de la calidad de la investigación comunicativa en España, a razón, fundamentalmente, del nacimiento de agencias como Aneca (Agencia Nacional de Evaluación de la Calidad y Acreditación) y de sus homólogas en los ámbitos autonómicos. También es muy importante destacar el trabajo documental y bibliométrico vinculado a los principales indicadores de calidad de la investigación (esencialmente vinculados a revistas científicas), concretado por ejemplo en los indicadores IN-RECS ${ }^{10}$ o RESH. ${ }^{11}$

En lo que se refiere a la temática y metodología de las investigaciones, el trabajo de Castillo y Carretón (2010) vinculado a las revistas de comunicación en España ${ }^{12}$ señala la supremacía de los estudios vinculados al

10 Por cuenta del Grupo de Investigación EC3 de Evaluación de la Ciencia y de la Comunidad Científica de la Universidad de Granada.

11 A cargo del Grupo de Investigación EC3 de Evaluación de la Ciencia y de la Comunidad Científica de la Universidad de Granada y del Grupo EPUC del CSIC.

12 Los autores analizan, a partir de la técnica de análisis bibliométrico, las diez revistas con mayor índice de impacto en el ámbito de la comunicación según el indicador IN-RECS para el año 2008. 
periodismo, seguidos de los que se ocupan de analizar la responsabilidad de los medios. De igual manera, se constata, en este mismo estudio, las preferencias por el uso de metodologías cuantitativas, especialmente el análisis de contenido, seguido de la encuesta y en menor medida el análisis del discurso y los grupos focales de discusión. Además se comprueba un dato revelador: en el $24 \%$ de los artículos de investigación analizados no se utiliza metodología alguna.

En una línea semejante se sitúa la investigación de Martínez-Nicolás y Saperas (2011). A partir de un análisis de contenido cualitativo de los artículos publicados en cuatro de las revistas de comunicación españolas de mayor impacto entre los años 1998 y 2007, los autores definen un mapa de investigación. El periodismo y la información periodística (prensa, radio, televisión y medios digitales) reúnen el 49,4\% de las investigaciones publicadas, seguidos de lejos por el ámbito de la comunicación audiovisual (17\%). Los contenidos representan el objeto de estudio preferido por la comunidad científica, seguidos del análisis de los profesionales y las empresas. En cuanto al tipo de investigación, predomina la empírica sin el uso de técnicas de investigación sistemáticas $(47,2 \%)$, seguida de la exclusivamente teórico-conceptual (21,3\%). Sorprende, de nuevo, que en el $62 \%$ de los artículos que incorporan investigación empírica no figure la utilización de ningún tipo de método/técnica, lo que denota, sin lugar a dudas, una preocupante carencia metodológica en la ejecución de investigaciones pretendidamente empíricas (Martínez-Nicolás 2006, 2009: Martínez-Nicolás y Saperas, 2011).

\section{La investigación en comunicación en Brasil}

En Brasil no existe una homogeneidad en la investigación en comunicación. Si se cruzan los datos de CNPq y de Fapesp. ${ }^{13}$ se encuentran grandes diferencias en cada uno de sus estados, donde en algunos casos el desarrollo es destacable (Río Grande del Sur o Río de Janeiro, con sobre el 25\% de la investigación), en otros muy destacado (São Paulo, con un 65\%) y en otros

13 Brasil tiene una agencia nacional de promoción de la investigación, el Conselho Nacional de Desenvolvimiento Científico e Tecnológico $(\mathrm{CNPq})$ y agencias estatales; la más importante de ellas es la Fundação de Amparo à Pesquisa do Estado de São Paulo (Fapesp). 
más se encuentran muchas carencias en el área (Río Grande del Norte o Bahía, con no más de un 10\%), considerando el 100\% de la investigación realizada en 2012 ( CNPq; Fapesp ${ }^{14}$ ). Las fuentes son tanto los proyectos de investigación aprobados en el área, las tesis doctorales leídas y las becas de postgrados como los informes de resultados de ambas agencias.

En el caso de São Paulo, por ejemplo, las cifras dan cuenta de que la investigación en comunicación, sólo en ese estado, es comparable con el resto de América Latina, excluyendo a México, y en algunos casos con cifras que superan la española. ${ }^{15}$ La Fundação de Amparo a Pesquisa do Estado de São Paulo (Fapesp) es una entidad que ha potenciado la investigación científica al ser una agencia que no depende de los gobiernos y contar con fondos permanentes obtenidos por la recaudación impositiva del estado.

Las temáticas de investigación en Brasil, por su parte estuvieron centradas, en un comienzo, fundamentalmente en la semiótica de la cultura, la teoría de la imagen visual, la cultura popular, la radio y la comunicación educativa. Más recientemente, se han incorporado la folk comunicación; la economía política, que ha adquirido un destacado impulso; la comunicación y la cultura y la comunicación y los medios (aunque en Brasil cuando se habla de medios, en plural, se están refiriendo a inter- o transmedios). Como indica Baitello Jr. (2006), la influencia anglosajona se hace presente hasta en el significante: mídia, incorporado del inglés media. Aunque mídia va más allá de medios y se refiere a una amplitud de significados, precisa Baitello (2006 y 2010), quien viene desde hace tiempo proponiendo las bases culturales de una teoría da mídia.

El investigador brasileño que ha tenido mayor visibilidad en América Latina es José Marques de Melo, quien pasó de lecturas de corte histórico y epistemológico (debate sobre la posibilidad de transformar la comunicación en una ciencia autónoma) a la incorporación de la folk comunicación. En uno de sus últimos escritos, "La investigación de la comunicación, ayer y hoy. Reto de la investigación latinoamericana en comunicación”, vuelve

14 Conselho Nacional de Desenvolmimento Cientifico e Tecnologico. Fundação de Amparo a Pesquisa do Estado de São Paulo (Fapesp). http://www.fapesp.br [fecha de consulta: 22 de enero de 2014].

15 Norval Baitello Jr., director del área comunicación de Fapesp 2014. Entrevista realizada para este artículo. 
a preguntarse sobre los retos de la investigación en comunicación en América Latina, indicando que "La nueva generación que se inicia en el campo de las ciencias de la comunicación, en este contexto de transición de milenio, sufre con la enfermedad que asola nuestras universidades, teniendo que superar la muralla de la amnesia histórica para no sucumbir delante del apego seductor de los que proclaman el 'fin de la historia' y la 'muerte de la utopía” (2007, p. 8).

Para entender los "desafíos actuales de esa área del conocimiento, en Latinoamérica, se torna indispensable rescatar su trayectoria intelectual para mejor percibir sus compromisos históricos. Ese retroceso puede ser hecho en tiempos distintos: el pasado reciente, distante y remoto" (Marques de Melo, 2007). Es decir, junto con otros teóricos vinculados a la economía política de la comunicación y la cultura, entre los que se encuentra otro brasileño, el investigador César Bolaño, recomienda volver a repasar la historia de la "rica escuela latinoamericana de la comunicación" y los antecedentes que la vinculan a asociaciones como Alaic, Felafacs y Ciespal, entre otras. En el caso de Brasil, además, otro aspecto que hay que destacar es el desarrollo de la semiótica y la teoría de la imagen.

Acentuado es el papel de la Associação Nacional de Programas de Pós-Graduação em Comunicação de Brasil (Compos), en cuyo sitio web, www.compos.org.br, se encuentra un histórico de relevancia sobre la investigación en comunicación en ese país. Compos se ha abierto a otras áreas de investigación en comunicación, como ciudadanía, cibercultura, experiencia estética, política e imaginarios mediáticos, diversificando los espacios y cuestionando, en este caso, la autonomía de los campos científicos.

Compos tiene como objetivos principales el fortalecimiento y la cualificación creciente de la posgraduación en comunicación, así como el estímulo y la participación de la comunidad académica, defendiendo el perfeccionamiento profesional y el desarrollo teórico, cultural, científico y tecnológico. En Compos, como en el caso de teóricos como Fuentes, se sigue hablando de campo de investigación, no obstante, por lo indicado la terminología epistémica debería de corregirse por la diseminación de la in- 
vestigación, la que da cuenta de un área en crecimiento posautónoma, transmedial y transdisciplinaria.

De los temas de investigación y publicaciones hay que destacar que en Brasil más del 50\% de la investigación se refiere a medios e inter-y transmedios, entre 42 revistas científicas y libros consultados. La televisión ocupa un lugar destacado seguida por la radio, entre otros aspectos, por la influencia de la Rede Globo, una de las seis cadenas de televisión más importantes del mundo.

En el caso de la radio, este medio tiene una influencia fundamental en megalópolis como São Paulo (como investiga Eugenio de Oliveira Menezes, 2007), ya que reduce la pesada carga de las largas jornadas en la ciudad. En la megalópolis se puede tardar en promedio unas seis horas en circular entre ella. Son destacadas las investigaciones sobre cibermedios y cultura digital (sobre el 30\% de la investigación total en comunicación presentada en 2012), tras consultar a Compos, CNPq y Fapesp.

En publicaciones hay que destacar el portal de Scielo (http://www. scielo.org), sistema de indexación creado por Fapesp que ha logrado sistematizar las revistas científicas que superan los 36 requisitos Latindex (en éste, con el cumplimiento de 34 requisitos ya es indexada la publicación; en Scielo los requisitos son más exigentes) para incorporarse. En Scielo Brasil, después del campo de la salud, es el de comunicación, ciencias de la información y tecnologías el que tiene la mayor cantidad de revistas indexadas, lo que da cuenta del crecimiento del área. Cabe destacar, a su vez, la Sociedade Brasileira de Estudos Interdisciplinares da Comunicação (Intercom), que se reúne en congresos periódicos para analizar, debatir y construir, desde la diversidad, un espacio de consolidación de la comunicación.

\section{Investigación en comunicación en Chile}

En Chile, a diferencia de otros países iberoamericanos, se ha intentado homogenizar la investigación científica en torno a una agencia de promoción y fomento: la Comisión Nacional de Investigación Científica y Tecnológica (Conicyt) y sus programas de fomento: Fondecyt, Fondef y becas de posgraduación (másters, doctorados y posdoctorados). 
Comunicación se encuentra junto con sociología en el área de ciencias sociales y humanidades y en el subárea de sociología y ciencias de la información. Del concurso regular de investigación, en el periodo de 2012 a 2013: de los 1135 concursados en total se aprobaron 631, de los cuales concursaron 52 y se obtuvieron 25 en la subárea de sociología y ciencias de la información.

En el histórico, analizando el período 2008 a 2011, las ciencias sociales, por detrás de las ciencias naturales, sigue siendo la segunda área con la mayor cantidad de proyectos aprobados, con un promedio de $30 \%$ anual. En 2008 se aprobó, en total, un $47 \%$ de los proyectos presentados, 260 de ellos en ciencias sociales; en el 2009, un $41 \%$ en total, 252 en el área; en 2010 , un $40 \%$, y bajaron a 67 en las disciplinas sociales, y en 2011, un 50\% en total, y el número subió a 96 (41\%) en las ciencias sociales (Fondecyt).

El periodismo sigue siendo el espacio de investigación más destacado y el más fomentado, con más de un $60 \%$ de presencia en la investigación en comunicación en el $100 \%$ de la investigación realizada en 2012. No obstante, han ido creciendo los proyectos sobre inter- $y$ transmedios (cultura digital, estudios visuales, redes digitales). Recientemente se han incorporado otras temáticas como la interculturalidad, inicialmente más vinculada a lo étnico y hoy abriéndose a los fenómenos migratorios.

En Chile, cabe destacar, es reciente la incorporación de la comunicación a los organismos de fomento de la investigación científica. A esto se suma la creación de la Asociación de Investigadores en Comunicación en 2011 para intentar conseguir mayores fondos para la investigación en comunicación en el conjunto de las ciencias sociales.

\section{Discusión y conclusiones}

El eterno debate que ocupa la discusión epistemológica y disciplinar en torno a los estudios de comunicación sigue vigente en nuestros días. La aparente 'solución' aportada por Bordieu y su 'teoría de los campos' parece no alcanzar la era de la posautonomía, caracterizada por un continuo mestizaje entre comunicación y arte, comunicación y desarrollo o comunicación y cultura, por citar sólo algunos ejemplos. La brecha abierta por la tecnología 
ha favorecido una cierta dilución de la teoría de los campos y, por ende, de su tan anunciada autonomía. De esta forma, los científicos pierden, de algún modo, el monopolio del conocimiento y la opinión en comunicación, que tiende cada vez más hacia un conocimiento menos institucionalizado y más popular. Ello supone cuestionar de nuevo la identidad epistemológica y disciplinar de la comunicación, pero esta vez desde un prisma diferente, priorizando el '¿hacia dónde vamos?' por delante del eterno '¿qué somos?’.

Asimismo, surge un nuevo e interesante debate en torno a la fijación epistemológica de la comunicación. A lo largo del texto, se han mostrado aportaciones relevantes en torno a este debate, como por ejemplo la de Arias y Roncallo (2012), quienes sugieren un enfoque más político (con relación al contexto, a la producción de subjetividades y en torno al apriorismo 'lo mío y lo de los otros' y, por consiguiente, en la definición del entre) del campo de la comunicación nuevamente centrado en analizar las condiciones de producción de una idea particular de unidad, el tipo de narrativas con las cuales han sido producidos y el por qué.

De igual forma, la investigación de la comunicación en España abandona, aunque sea parcialmente, el protagonismo de los medios de comunicación masivos, para centrar parte de los nuevos intereses de investigación en la convergencia mediática, las nuevas pantallas o el universo digital transmediático. Si bien aumenta la producción científica en comunicación, también es cierto que esa misma investigación continúa incorporando importantes carencias (sobre todo a nivel metodológico) con las cuales se deberá seguir trabajando en el futuro. Aun así, la aparición de indicadores de calidad de la investigación, la creación en $2006^{16}$ de la Asociación Española de Investigación de la Comunicación (AE-IC),${ }^{17}$ el surgimiento de agencias de evaluación cualitativa de esa misma investigación y de sus investigadores, y el reconocimiento internacional que progresivamente acumulan algunas publicaciones científicas dedicadas a la comunicación en España parecen au-

16 La asamblea fundacional de la asociación tuvo lugar el 14 de noviembre de 2006, mientras que el congreso fundacional se celebró en Santiago de Compostela en 2008 (www.ae-ic.org, 23 de diciembre de 2012).

17 La Asociación Española de Investigación de la Comunicación (AE-IC) tiene como misión potenciar la investigación de la comunicación en el conjunto de la política científica, facilitar la cooperación y crear redes de intercambio de información entre investigadores, y establecer una política de cooperación con asociaciones internacionales de investigación (www.ae-ic.org, 3 de enero de 2013). 
gurar un futuro sin duda más exigente con el cientificismo comunicativo, lo que debería incidir en una clara mejora de la repercusión internacional de la investigación en comunicación que actualmente se desarrolla y en el futuro se llevará a cabo en España.

En la posmodernidad se da un intento por alejarse de los datos empíricos y la comunicación; en muchos momentos, esta tendencia sigue, lo que debilita su espacio de influencia en el conjunto de las ciencias sociales. Por ello, no existen los datos definitivos -como pudimos comprobar en esta investigación-, sino la posibilidad de ir diseñando mapas iniciales que permitan a largo plazo tener una visión más acabada de la investigación en comunicación.

En el caso de Brasil, la diseminación es frecuente y, a excepción de estados como São Paulo, no hay datos definitivos; no obstante, se está intentando consolidar la comunicación como espacio emergente pero, paralelamente, fructífero al interior de la investigación científica. Un tercer país emergente, como Chile, ha ido homogeneizando la investigación científica y recientemente incorporó la comunicación en el conjunto de las ciencias sociales, transformándola rápidamente en una de las áreas con mayor crecimiento.

\section{Referencias}

Arias, J. C. y Roncallo, S. (2012). "Reordenando la reflexión. De la epistemología a la política. Algunas preguntas sobre el campo de la comunicación”. En: Logos, 21, pp. 213-228.

Bachelard, G. (1972). La formación del espíritu científico. Buenos Aires: Siglo XXI.

Baitello Jr., N. (2006). La era de la iconofagia. Sevilla: Arcibel.

Baitello Jr., N. (2010). A serpente, a maçã e o holograma. Esboços para uma Teoria da Mídia. São Paulo: Paulus.

Bourdieu, P. (2000). Los usos sociales de la ciencia. Buenos Aires: Claves. 
Cáceres, M. D. y Caffarel, C. (1992). "La comunicación en España: planteamientos temáticos y metodológicos entre 1987 y 1990”. En: A.I.C.E. (ed.). La investigación en la comunicación (pp. 23-30). Madrid: A.I.C.E.

Cáceres, M. D. y Caffarel, C. (1993). "La investigación sobre comunicación en España. Un balance cualitativo”. En: Telos, 32, pp. 1-12.

Castillo, A.y Xifra, J. (2006). “Investigación bibliométrica de las tesis doctorales españolas sobre relaciones públicas (1965-2005)”. En: Anàlisi, 34, pp. 141-161.

Castillo, A. y Carretón, M.C. (2010). "Investigación en Comunicación. Estudio bibliométrico de las revistas de comunicación en España”. En: Comunicación y Sociedad, 23 (2), pp. 289-327.

Conselho Nacional de Desenvolvimento Científico e Tecnologico (CNPq). http://www.cnpq.br [fecha de consulta: 22 de enero de 2014]

Craig, R. T. (1999). “Communication Theory as a Field”. En: Communication Theory, 9 (2), pp. 119-161.

De Aguilera, M. (1998). "La investigación sobre comunicación en España: una visión panorámica”. Comunicación y Cultura, 4, pp. 5-11.

Del Rey Morató, J. (1997). “No usarás el nombre de la ciencia en vano. Manifiesto por la Universidad del siglo XXI. Carta abierta a los jóvenes investigadores y a los investigadores no tan jóvenes”. En: Cuadernos de Información y Comunicación, 3, pp. 47-80.

Donsbach W. (2006). “The Identity of Communication Research”. En: Journal of Communication, 56 (1), pp. 437-448.

Dulzaides, M. E. y Molina, A. M. (2004). "Análisis documental y de información: dos componentes de un mismo proceso". En: ACIMED, 2 (12), pp. 1-4. 
Fondo Nacional de Desarrollo Científico y Tecnológico (Fondecyt).http:// www.fondeyct.cl [fecha de consulta: 20-24 de marzo de 2014].

Foucault, M. (1986). La arqueología del saber. México: Siglo XXI.

Fuentes, R. (2008). La comunicación desde una perspectiva sociocultural. Acercamientos y provocaciones 1997-2007. Guadalajara: Iteso.

Fuentes, R. (2012). "Prefacio". En: Rizo, M. (ed.). Imaginarios sobre la comunicación. Algunas certezas y muchas incertidumbres en torno a los estudios de comunicación, hoy (pp. 13-15). Bellaterra: Universitat Autònoma de Barcelona.

Fundação de Amparo a Pesquisa do Estado de São Paulo (Fapesp). http:/ / www.fapesp.br [fecha de consulta: 22 de enero de 2014].

García Canclini, N. (2011). La sociedad sin relato. Antropología y estética de la inminencia. Barcelona: Katz.

García-Jiménez, L. (2007). Las teorías de la comunicación en España: un mapa sobre el territorio de nuestra investigación (1980-2006). Madrid: Tecnos.

Jones, D. E. (1998). “Investigación sobre comunicación en España: evolución y perspectivas”. Zer, 3, pp. 13-51.

Kuhn, T. (1981). Las estructuras de las revoluciones cientificas. México: F. C.E.

Marques de Melo, J. (2007). "La investigación de la comunicación, ayer y hoy. Reto de la investigación latinoamericana en comunicación”. En: Chasqui, 100, pp. 8-13.

Martínez-Nicolás, M. (2006). "Masa (en situación) crítica. La investigación sobre periodismo en España: comunidad científica e intereses de conocimiento". En: Anàlisi, 33, pp. 135-170. 
Martínez-Nicolás, M. (2009). "La investigación sobre comunicación en España. Evolución histórica y retos actuales”. En: Revista Latina de Comunicación Social, 64, pp. 1-14.

Martínez-Nicolás, M. y Saperas, E. (2011). "La investigación sobre comunicación en España (1998-2007). Análisis de los artículos publicados en revistas científicas". En: Revista Latina de Comunicación Social, 66, pp. 1-29.

Moragas, M. de (2011). Interpretar la comunicación. Estudios sobre medios en América y Europa. Barcelona: Gedisa.

Oliveira Menezes, J. E. (2007). Rádio e cidade: vínculos sonoros. São Paulo: Annablume.

Pereira, J. M. (2005). “La comunicación: un campo de conocimiento en construcción. Reflexiones sobre la comunicación social en Colombia”. En: Investigación y Desarrollo 13 (2), pp. 412-441.

Piñuel, J. L. (2010). "La comunicación como objeto científico de estudio, campo de análisis y disciplina científica”. En: Contratexto, 18, pp. 67-107.

Rizo, M. (2012). Imaginarios sobre la comunicación. Algunas certezas y muchas incertidumbres en torno a los estudios de comunicación, hoy. Bellaterra: Universitat Autònoma de Barcelona.

Rodrigo, M. (2001). Teorías de la comunicación. Ámbitos, métodos y perspectivas. Barcelona: Aldea Global.

Sheperd, G. J. (1993). “Building a Discipline of Communication”. En: Journal of Communication, 43 (3), pp. 83-91.

Silva, V. y Browne, R. (2007). Antropofagias. Las indisciplinas de la comunicación. Madrid: Biblioteca Nueva. 